\title{
Ionic liquid pre-intercalated MXene films for ionogel-based flexible micro-supercapacitors with high volumetric energy density
}

\author{
Shuanghao Zheng, $\ddagger_{a b c}$ Chuanfang (John) Zhang, $\ddagger_{d}$ Feng Zhou,a Yanfeng Dong, Xiaoyu Shi,abe Valeria Nicolosi, ${ }^{1}$ Zhong-Shuai Wu \\ (D) $*_{a}$ and Xinhe Bao $\mathbb{D}_{a b}$
}

\begin{abstract}
MXenes are emerging as a very promising electrode material of highperformance micro-supercapacitors (MSCs) for microscale electronics. However, MXene based MSCs (M-MSCs) reported so far exhibit low areal $\left(\# 10 \mathrm{~mW} \mathrm{~h} \mathrm{~cm}{ }^{2}\right.$ ) and volumetric $\left(\# 20 \mathrm{~mW} \mathrm{~h} \mathrm{~cm}{ }^{3}\right.$ ) energy densities due to their narrow working voltage in aqueous systems (0.6-1.0 V). Herein, we report for the first time the construction of high-voltage and high-energy flexible ionogel-based M-MSCs with interdigital microelectrodes of ionic liquid preintercalated MXene films. Benefitting from the pre-intercalation of ionic liquid, the as-fabricated M-MSCs, working at $3 \mathrm{~V}$ in 1-ethyl-3methylimidazolium tetrafluoroborate $\left(\mathrm{EMIMBF}_{4}\right)$, exhibited high areal and volumetric energy densities of $13.9 \mathrm{~mW} \mathrm{~h} \mathrm{~cm}^{2}$ and 43.7 $\mathrm{mW} \mathrm{h} \mathrm{cm}{ }^{3}$, respectively, both of which are among the highest values for the reported M-MSCs. Moreover, all-solid-state M-MSCs using ionogel electrolytes displayed exceptional flexibility without capacitance loss under various deformation conditions and seamless integration free of metal-based interconnections for boosting voltage output. Therefore, such high-energy M-MSCs hold great potential for direct integration of flexible and miniature electronics.
\end{abstract}

The dramatic development of wearable and miniature electronics, e.g., microelectromechanical systems, microrobots, and microsensors, has greatly accelerated the exploitation of highperformance microscale electrochemical energy storage units that are small and lexible enough to be strongly coupled with such electronics. ${ }^{1-6}$ Recently, micro-supercapacitors (MSCs), with in-plane microelectrode geometry on a single substrate, have been gradually acknowledged as a novel class of highly competitive miniaturized energy storage devices, ${ }^{7-9}$ possessing remarkable merits of ultrafast charge-discharge rate, ultrahigh power density, long-term cyclability, outstanding safety, and being separatorfree. ${ }^{2-13}$ To date, great efforts have been concentrated on the performance tailoring of planar MSCs based on different electrode materials, for instance, nanocarbons (e.g., carbide derived carbon, ${ }^{14}$ onion-like carbon, ${ }^{15}$ carbon nanotubes, ${ }^{16}$ and graphene ${ }^{17-20}$ ), MXenes, ${ }^{21-24}$ conducting polymers, ${ }^{25,26}$ and metal oxides ${ }^{27}$ and hydroxides. ${ }^{28}$ Among them, atomically thin two-dimensional (2D) materials with an intrinsically ?at and ?exible structure can match well with the in-plane geometry of MSCs, in which the electrolyte ions are capable of fully utilizing the lat architecture and ultrathin thickness of 2D materials and realizing fast ionic diffusion along the plane direction of $2 \mathrm{D}$ nanosheet-based nanochannels. ${ }^{29}$ Consequently, graphene and other analogous 2D nanosheets are regarded as promising electrode materials for the creation of planar MSCs. ${ }^{30}$ However, both the areal and volumetric energy densities of most reported 2D nanosheetbased MSCs still fail to compete with those of commercially available lithium thin-? $1 \mathrm{~m}$ microbatteries $\left(\# 10 \mathrm{~mW} \mathrm{~h} \mathrm{~cm}{ }^{3}\right)$ for miniaturized electronics and self-powered microsystems. 15

MXenes are an emerging large family of 2D early transition metal carbides and carbonitrides from the 60+ group of MAX phases, with engineered surface chemistry and exceptional ion intercalation behavior, having tremendous potential for numerous energy storage devices from batteries to supercapacitors. ${ }^{31-39} \mathrm{In}$ particular, $\mathrm{Ti}_{3} \mathrm{C}_{2} \mathrm{~T}_{\mathrm{X}}$ is the most widely studied MXene for supercapacitors with an ultrahigh volumetric capacitance up to $1500 \mathrm{~F} \mathrm{~cm}^{3}$ and ultrahigh electrical conductivity (9800 S cm$)^{1}$ ), outperforming most reported electrode materials.40-42 Unfortunately, high volumetric capacitance of such $\mathrm{Ti}_{3} \mathrm{C}_{2} \mathrm{~T}_{\mathrm{X}} \mathrm{MXene}$ based MSCs (M-MSCs) is normally obtained in aqueous electrolytes, with a very limited cell voltage of $0.6-1.0 \mathrm{~V}$ in a symmetric con? louration. Therefore, the resulting MSCs exhibited low areal (\#10 $\left.\mathrm{mW} \mathrm{h} \mathrm{cm}^{2}\right)$ and volumetric $\left(\# 20 \mathrm{~mW} \mathrm{~h} \mathrm{~cm}{ }^{3}\right.$ ) energy density. ${ }^{22,24,43,44}$ On the other hand, using an ionic liquid as the electrolyte can signi国antly broaden the voltage window of supercapacitors, but two reported MXene based cells operated at $3 \mathrm{~V}$ so far still offer low gravimetric capacitance ( $80 \mathrm{~F} \mathrm{~g}^{1}$ ) and unsatisfactory cycling stability. ${ }^{45,46}$ To realize high-density electrochemical capacitive energy storage, Li's group developed a general strategy to create liquid electrolyte-mediated graphene compact ?lms, with a high ion-accessible surface area and low ion transport resistance, for boosting the volumetric energy density of supercapacitors to $60 \mathrm{~W} \mathrm{~h} \mathrm{~L}{ }^{1}{ }^{47}$ Nevertheless, rational design and construction of ionic liquid mediated MXene ?llms for high energy MSCs remains undeveloped.

Here, we reported the on-chip fabrication of a new type of high-voltage ionogel-based MSC with interdigital microelectrode ?ngers of ionic liquid pre-intercalated MXene ?lms, simultaneously achieving high volumetric energy density, exceptional ?exibility and modular integration of bipolar cells. With interdigital mask-assisted deposition of MXene and graphene, the resulting interdigital microelectrodes possessed an

\footnotetext{
${ }^{1}$ CRANN, AMBER, School of Chemistry, Trinity College Dublin, Dublin 2, Ireland e Department of Chemical Physics, University of Science and Technology of China, 96 JinZhai Road, Hefei 230026, China

† Electronic supplementary information (ESI) available. See DOI:

${ }^{2} .1039 / \mathrm{c} 9 \mathrm{ta} 02190 \mathrm{f}$

¥ These authors contributed equally to this work.
} 
outstanding electrical conductivity of $2200 \mathrm{~S} \mathrm{~cm}^{1}$ without a polymer binder and conductive additives as well as metal current collectors. Bene?tting from the pre-intercalation of ionic liquids, MXene based electrode ?lms continuously developed an ion transport network with an expanded interlayer spacing of $1.45 \mathrm{~nm}$, which is larger than that of the fresh undried MXene (1.27 nm) and fully dried MXene (1.09 $\mathrm{nm})$. The resulting MSCs, assembled with the EMIMBF 4 pre-intercalated lllms and tested in the same electrolyte of EMIMBF 4 , delivered a remarkably high volumetric capacitance of $140 \mathrm{~F} \mathrm{~cm}^{3}$, high areal energy density of $13.9 \mathrm{~mW} \mathrm{~h} \mathrm{~cm}{ }^{2}$ and volumetric energy density of $43.7 \mathrm{~mW} \mathrm{~h} \mathrm{~cm}^{3}$, the latter two of which are the highest values of the state-of-the-art M-MSCs reported. Notably, all-solid-state MSCs using an ionogel electrolyte composed of $\mathrm{EMIMBF}_{4}$ and poly(vinylidene ?uoridehexa?luoropropylene) (EMIMBF $\left.4 / \mathrm{PVDF}-\mathrm{HFP}\right)$ also exhibited a high areal energy density of $13.3 \mathrm{~mW} \mathrm{~h} \mathrm{~cm}^{2}$ and volumetric energy density of $41.8 \mathrm{~mW} \mathrm{~h} \mathrm{~cm}^{3}$, accompanied by long-term cyclability up to 10000 cycles. Furthermore, our ionogel based MSCs showed outstanding lexibility without capacitance degradation under repeated deformation conditions, and are readily integrated without any requirement of metal interconnection to increase output voltage and capacitance.

The fabrication of M-MSCs is schematically elucidated in Fig. 1a. Typically, four steps were involved. Firstly, the interdigital patterns of MXene microelectrodes $\left(1.6 \mathrm{~mL}, 0.65 \mathrm{mg} \mathrm{mL}^{1}\right)$ were created with the assistance of an interdigital mask.

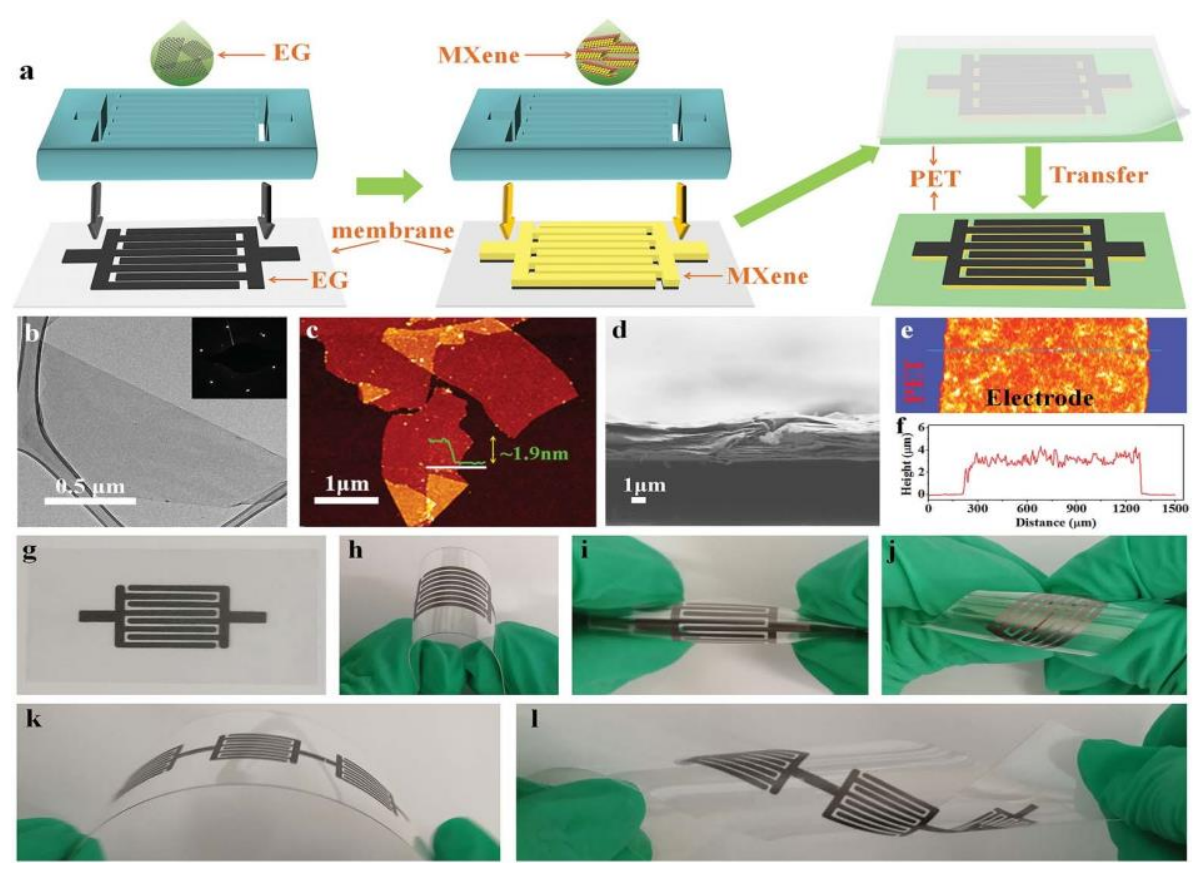

Fig. 1 Fabrication and characterization of M-MSCs. (a) Schematic of the fabrication of M-MSCs. (b) TEM image of MXene. The inset shows a selected area electron diffraction image. (c) Atomic force microscopy image of single-layer MXene nanosheets. The inset shows the height profile. (d) Cross-sectional SEM image and (e) surface profile map of a microelectrode finger. (f) Height profile of a microelectrode finger of MMSCs. (g-j) Photographs of M-MSCs in flat (g), bending ( $\mathrm{h}$ and $\mathrm{i}$ ) and twisting (j) states. ( $\mathrm{k}$ and $\mathrm{l}$ ) Photographs of three serially connected M-MSCs in bending (k) and spiral (I) states. 
The ultrathin $\mathrm{Ti}_{3} \mathrm{C}_{2} \mathrm{~T}_{\mathrm{X}}$ MXene nanosheets, with a typical lateral size of 1-3 mm (Fig. 1b) and single-layer thickness of $1.9 \mathrm{~nm}$ (Fig. $1 \mathrm{c}$ ), ${ }^{48}$ were prepared by etching the $\mathrm{Ti}_{3} \mathrm{AlC}_{2} \mathrm{MAX}$ phase with a $\mathrm{LiF} / \mathrm{HCl}$ solution. ${ }^{24,39,49} \mathrm{It}$ is worth noting that, to construct the highly uniform $\mathrm{MXene}$ ? $1 \mathrm{~m}$ and ensure the following full transfer of microelectrodes from the ?ltration membrane onto the lexible substrate, we selected high-quality, large-size and solution-processable electrochemically exfoliated graphene (EG, $2 \mathrm{~mL}, 0.1 \mathrm{mg} \mathrm{mL}{ }^{1}$, Fig. S1†) as the bottom-layer ?exible elastic support on a polyvinylidene ?uoride (PVDF) membrane, which is critical for uniform deposition of MXene ?lms. Secondly, the resulting patterned MXene-based microelectrodes were directly transferred onto a lexible polyethylene terephthalate (PET) substrate under 20 MPa pressure. Without the bottom EG layer, the interdigital MXene layer readily suffered from short circuit. Bene?tting from the metallic conductivity of MXene nanosheets, the microelectrodes exhibited a high electrical conductivity of $2200 \mathrm{~S} \mathrm{~cm}^{1}$ (Fig. S2 + ), enabling fast electron transport without the need for a metal-based current collector. Cross-sectional scanning electron microscopy (SEM) images (Fig. 1d and S3 + ) display the uniform layer-stacked structure of microelectrode ?lms, which is highly favorable for the fast transport of ions along the lat plane of MXene nanosheets. The surface pro?le (Fig. 1e) revealed the large-scale ?at uniformity of the microelectrode ?ngers, with a stacking density of $2.1 \mathrm{~g} \mathrm{~cm}^{3}$ and an average thickness of $3.2 \mathrm{~mm}$ (Fig. 1f and S4†). Remarkably, the obtained M-MSCs (Fig. 1g) exhibited excellent lexibility without any fragmentation of electrode ? lms and delamination between microelectrode ? llms and the PET substrate under severe deformation conditions, e.g., serious bending (Fig. 1h and i) and twisting (Fig. 1j) states.

Moreover, integrated M-MSCs connected in series or in parallel, such as three serially connected M-MSCs in bending (Fig. 1k) and spiral (Fig. 11) states, can be readily realized through a one by one transfer strategy, and displayed extraordinary lexibility without any degradation.
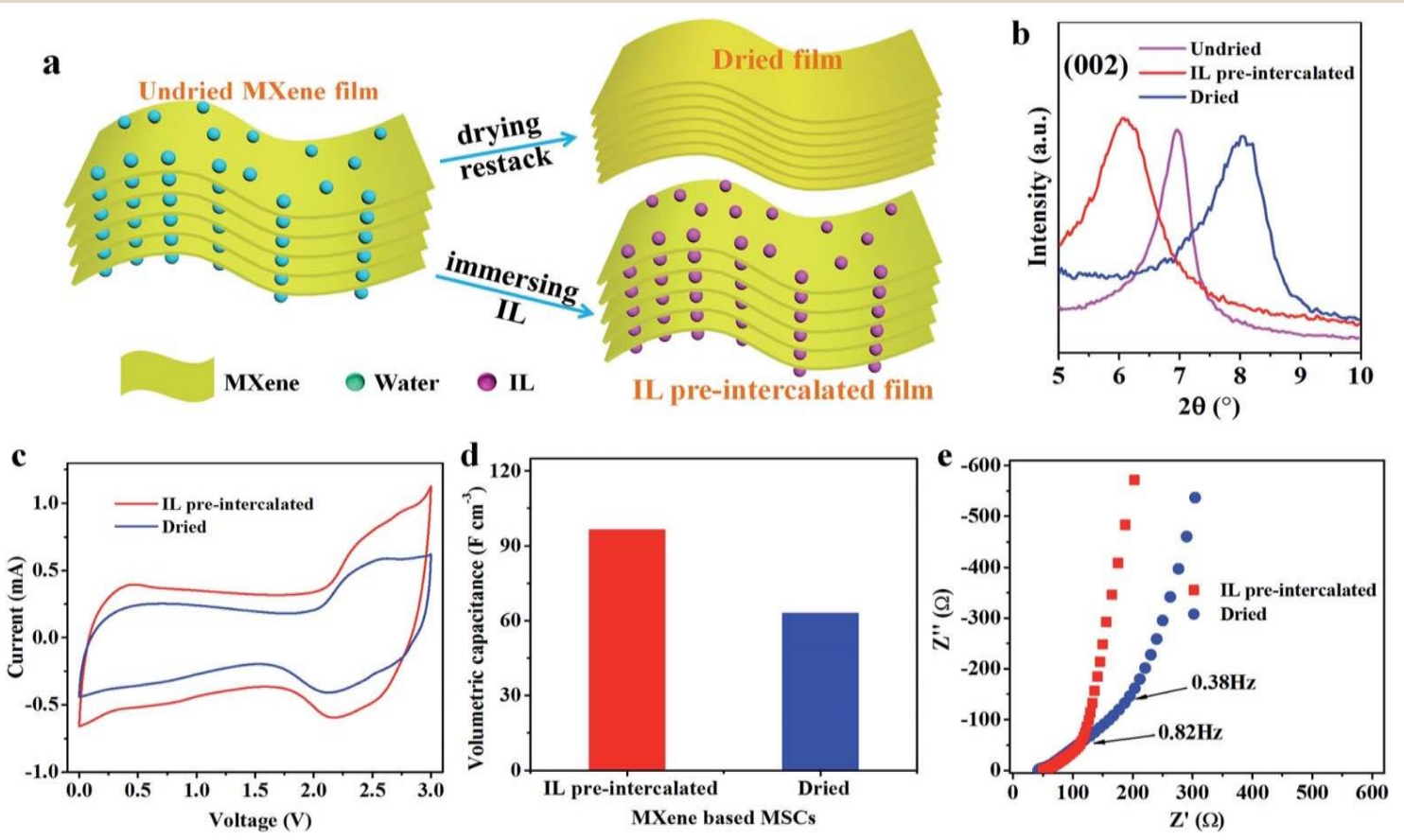

Fig. 2 Comparison of $\mathrm{EMIMBF}_{4}$ pre-intercalated and dried MXene films for M-MSCs, tested in EMIMBF 4 . (a) Schematic of the preparation of ionic liquid (IL) preintercalated and dried MXene electrode films. (b) XRD patterns of IL pre-intercalated and dried MXene films in comparison with that of the freshly undried film. (c) CV curves tested at $50 \mathrm{mV} \mathrm{s}^{1}$, (d) volumetric capacitance, and (e) EIS spectra of M-MSCs based on an IL preintercalated film and a dried MXene film.

Thirdly, the obtained fresh undried microelectrodes were completely immersed into the test ionic liquid electrolytes (e.g., EMIMBF 4 ) for $24 \mathrm{~h}$

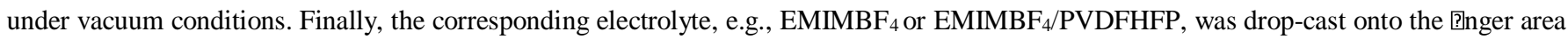
of interdigital microelectrodes, and high-voltage ionic liquid or ionogel based MMSCs were achieved.

To highlight the importance of ionic liquid pre-intercalation into the MXene lllm for the performance improvement of MMSCs, we fabricated two kinds of M-MSCs, one is denoted as PM-MSCs based on the ionic liquid pre-intercalated MXene (PM) [?lm a? immersion in $\mathrm{EMIMBF}_{4}$ for $24 \mathrm{~h}$, and the other is denoted as DM-MSCs based on the fully dried MXene (DM) alm at $120 \mathrm{C}$ for $24 \mathrm{~h}$ under vacuum for comparison. As expected, X-ray diffraction (XRD) patterns (Fig. 2b and S5†) disclosed that the (002) diffraction peak of the PM

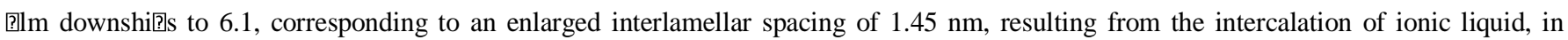




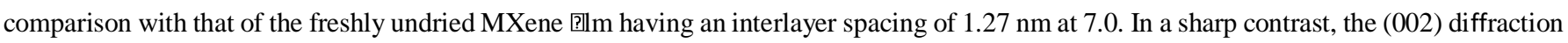
peak of the DM ? MXene ? $\mathrm{lm}$. Note that the vacuum-annealing treatment didn't induce the oxidation of MXene @llms, as demonstrated from Raman spectra (Fig. S6+).

To further verify this disparity, the electrochemical performances of PM-MSCs and DM-MSCs were evaluated in $\mathrm{EMIMBF}_{4}$ at a voltage of $3 \mathrm{~V}$, tested at $50 \mathrm{mV} \mathrm{s}^{1}$. Apparently, the cyclic voltammetry (CV) curve of PM-MSCs exhibited a larger integration area than that of DMMSCs (Fig. 2c), indicative of the enhanced capacitance of PM-MSCs. It can be observed that the broad redox peaks, appearing at 2.0-2.5 V, are primarily attributed to the pseudocapacitive intercalation/deintercalation behavior of $\mathrm{EMIM}^{+}$cations along the interlayer nanochannels of MXene [llms. ${ }^{50}$ Signi?cantly, PM-MSCs delivered a volumetric capacitance of $96 \mathrm{~F} \mathrm{~cm}^{3}$, which is 1.5 times higher than that of DM-MSCs (63 $\mathrm{F} \mathrm{cm}^{3}$ ) at $50 \mathrm{mV} \mathrm{s}^{1}$. It can be noted that the capacitance contribution of the thin EG layer to the total capacitance of PM-MSCs is negligible, about $1.2 \%$ (Fig. S7†). Furthermore, the electrochemical impedance spectrum (EIS) of PM-MSCs displayed a higher knee frequency of 0.82 $\mathrm{Hz}$ and larger slope in the low frequency range (Fig. 2e), in comparison with that of DM-MSCs (0.38 Hz), re?lecting enhanced capacitive behaviors and fast ion dynamics of PM-MSCs. These results further demonstrate that the pre-intercalation of the ionic liquid electrolyte into MXene ?lms with expanded interlayer spacing can intensively accommodate more available ions, provide a high ion-accessible surface area and low ion transport resistance, and thus dramatically increase the capacitance of electrochemically active MXene materials. ${ }^{41,47}$ In addition, it should be mentioned that the water content in the ionic liquid electrolyte was about $0.6 \mathrm{wt} \%$, which didn't exert an effect on the stability of ionic liquids tested at $3 \mathrm{~V}$ (Fig. S8+). ${ }^{51}$ And this result was also demonstrated from the polarization curves of PM-MSCs (Fig. S9+).

To maximize the electrochemical performance of planar PMMSCs (Fig. 3a), we further optimized the ?lm thickness of MXene

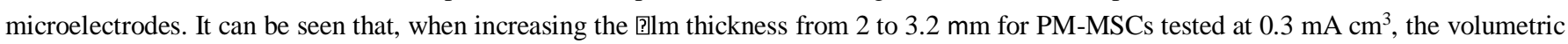
capacitance gradually increased from 98 to $122 \mathrm{~F} \mathrm{~cm}^{3}$ (Fig. 3b and S10†). The discharge times of PMMSCs based on the 3.2 and 3.6 mm-thick ⿴囗玉 $1 \mathrm{~ms}$ almost remained unchanged, which is likely ascribed to the inadequately preembedded electrolyte ions for thicker microelectrodes. Furthermore, we also investigated the effect of the type of preintercalated and tested ionic liquid on the performance of PM-MSCs, using the $3.2 \mathrm{~mm}$-thick MXene ? lms. Speci?leally, the four ionic liquids are EMIMBF4, 1-ethyl-3-methylimidazolium bis(tri?luoromethylsulfonyl)imide

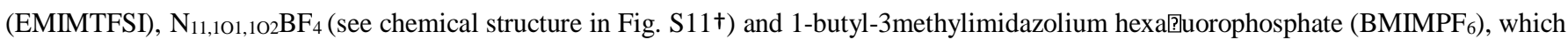
were correspondingly denoted as PM-MSCs-EB, PM-MSCs-ET, PM-MSCs-NB and PM-MSCs-BP, respectively. Both the CV curves obtained at $50 \mathrm{mV} \mathrm{s}^{1}$ (Fig. 3d) and the galvanostatic charge and discharge (GCD) profles measured at $0.4 \mathrm{~mA} \mathrm{~cm}^{2}$ (Fig. 3e) revealed that all the PMMSCs working in these ionic liquids can be well operated at $3 \mathrm{~V}$, exceeding those of aqueouselectrolyte based M-MSCs, for instance, $0.6 \mathrm{~V}$ in $1 \mathrm{M} \mathrm{H}_{2} \mathrm{SO}_{4},{ }^{34} 0.8 \mathrm{~V}$ in polyvinyl alcohol (PVA)/ $\mathrm{H}_{3} \mathrm{PO}_{4},{ }^{23}$ and $1.0 \mathrm{~V}$ in $3 \mathrm{M} \mathrm{H}_{2} \mathrm{SO}_{4} .{ }^{52}$ In comparison with PM-MSCs-ET, PM-MSCs-NB and PM-MSCs-BP, PM-MSCs-EB showed a larger integration area of the CV curve and longer discharge time in the GCD pro?le, indicative of the higher capacitance of PM-MSCs-EB. It should be pointed out that the redox peaks of PM-MSCs-EB and PMMSCs-ET resulted from the insertion and extraction of $\mathrm{EMIM}^{+}$cations during charge and discharge. ${ }^{46,53}$ As a result, PM-MSCsEB tested at a low current density of $0.1 \mathrm{~mA}$ $\mathrm{cm}^{2}$ delivered a high areal capacitance of $44 \mathrm{mF} \mathrm{cm}^{2}$ (Fig. 3f) and volumetric capacitance of $139 \mathrm{~F} \mathrm{~cm}^{3}$ (Fig. $3 \mathrm{~g}$ ), both of which are much higher than those of PM-MSCs-ET (29 $\mathrm{mF} \mathrm{cm}{ }^{2}$ and $\left.93 \mathrm{~F} \mathrm{~cm}^{3}\right)$, PM-MSCs-NB $\left(33 \mathrm{mF} \mathrm{cm} \mathrm{cm}^{2}\right.$ and $\left.103 \mathrm{~F} \mathrm{~cm}^{3}\right)$ and PM-MSCs-BP $\left(12 \mathrm{mF} \mathrm{cm}^{2}\right.$ and $39 \mathrm{~F}$ $\mathrm{cm}^{3}$ ). Moreover, PM-MSCs-EB, tested at a high current density of $1.0 \mathrm{~mA} \mathrm{~cm}{ }^{2}$, also presented excellent rate capability, with a considerable areal capacitance of $29 \mathrm{mF} \mathrm{cm}{ }^{2}$ and volumetric capacitance of $91 \mathrm{~F} \mathrm{~cm}^{3}$, outperforming PM-MSCs-ET $\left(19 \mathrm{mF} \mathrm{cm}^{2}\right.$ and $\left.60 \mathrm{~F} \mathrm{~cm}{ }^{3}\right)$, PMMSCs$\mathrm{NB}\left(13 \mathrm{mF} \mathrm{cm}{ }^{2}\right.$ and $\left.42 \mathrm{~F} \mathrm{~cm}^{3}\right)$ and PM-MSCs-BP $\left(3 \mathrm{mF} \mathrm{cm}^{2}\right.$ and $\left.8 \mathrm{~F} \mathrm{~cm}^{3}\right)$. This can be well explained by the lower equivalent series resistance (ESR) of PM-MSCs-EB (50 U) (Fig. 3h and S12†) and higher ionic conductivity of EMIMBF $4\left(14 \mathrm{mS} \mathrm{cm}^{1}\right.$, Fig. S13†), ${ }^{54}$ as compared with

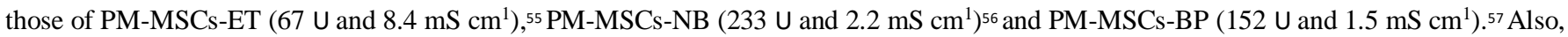
based on the frequency at the maximum imaginary speci]c capacitance $\left(\mathrm{C}^{00}\right)$, PM-MSCs-EB showed a high characteristic frequency of 0.15 $\mathrm{Hz}$ (Fig. S14t), demonstrative of the fast ion transfer of EMIMBF 4 between MXene nanosheets. In addition, PM-MSCs-EB exhibited outstanding cycling stability at $1.5 \mathrm{~mA} \mathrm{~cm}{ }^{2}$, with a capacitance retention of $92 \%$ afler 1000 cycles, much better than those of previously reported $\mathrm{Ti}_{3} \mathrm{C}_{2} \mathrm{~T}_{\mathrm{x}}$ ? $1 \mathrm{~lm}$ supercapacitors in EMIMTFSI ( $80 \%, 1000$ cycles). 45,46 

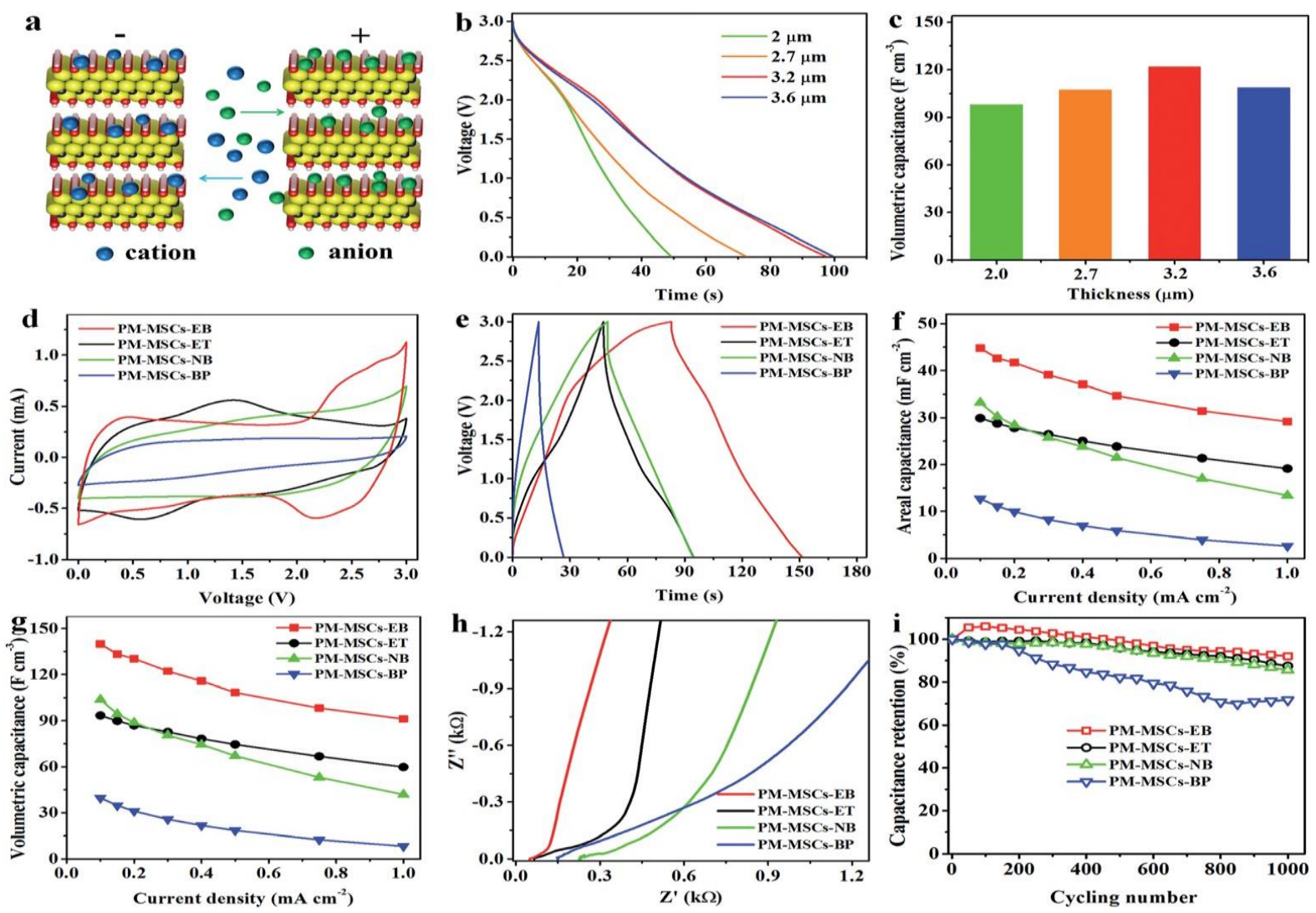

Fig. 3 Electrochemical performance of PM-MSCs measured in different ionic liquids. (a) Schematic diagram of parallel ion transfer between MXene nanosheet based films. (b) Discharge profiles and (c) volumetric capacitance of PM-MSCs-EB with various electrode thicknesses, tested at $0.3 \mathrm{~mA} \mathrm{~cm}^{3}$. (d) $\mathrm{CV}$ curves obtained at $50 \mathrm{mV} \mathrm{s}^{1}$, (e) GCD profiles measured at $0.4 \mathrm{~mA} \mathrm{~cm}^{2}$, (f) areal capacitance, (g) volumetric capacitance, (h) EIS spectra and (i) cycling stability of PMMSCs-EB, PM-MSCs-ET, PM-MSCs-NB and PM-MSCs-BP, respectively. 
To meet the booming demand for wearable, ?exible and integrated microelectronics, we further constructed all-solidstate PM-MSCs based on a microelectrode thickness of $3.2 \mathrm{~mm}$ and the EMIMBF $4 /$ PVDF-HFP ionogel electrolyte (denoted as PM-MSCs-EBIE, Fig. 4a), with an exceptional ionic conductivity of $25 \mathrm{mS} \mathrm{cm}{ }^{1}$ (Fig. S15+). ${ }^{19}$ From Fig. $4 \mathrm{~b}$ and c, it can be seen that both CV curves and GCD pro?les of PMMSCs-EBIE are largely similar to those of PM-MSCs-EB, suggestive of coherent electrochemical behavior. Notably, PM-MSCs-EBIE at a low current density of $0.1 \mathrm{~mA} \mathrm{~cm}{ }^{2}$ delivered areal (Fig. 4d) and volumetric (Fig. 4e) capacitances of $42 \mathrm{mF} \mathrm{cm}^{2}$ and $133 \mathrm{~F} \mathrm{~cm}$, respectively, both of which were slightly lower than those of PM-MSCs-EB (44 mF cm ${ }^{2}$ and $139 \mathrm{~F} \mathrm{~cm}^{3}$, Fig. 3f and g). At a high current density of $1 \mathrm{~mA}$

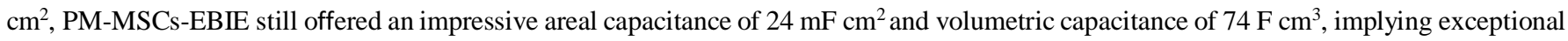
rate capability. Also, PM-MSCs-EBIE displayed a lower ESR of $45 \mathrm{U}$ compared to PM-MSCs-EB (50 U) because of the enhanced ionic conductivity of the ionogel electrolyte (Fig. S16+). Additionally, PM-MSCs-EBIE showed exceptional cyclability without capacitance degradation a? 10000 cycles at $1.0 \mathrm{~mA} \mathrm{~cm}$ (Fig. 4f). The initial increase of capacitance is mainly attributed to the gradual increase in the ion accessible area of MXene [lms originating from the slow in ?ltration of the ionogel electrolyte. With cycling, more and more ions were gradually inserted into the inner of MXene microelectrodes, leading to the gradual increase of capacitance. It is highlighted that such a longlife cyclability of MXene based supercapacitors in ionic liquid has never been achieved. To verify the ?exibility of the as-fabricated microdevices, we further examined the CV curves of PM-MSCsEBIE in different bending states (Fig. 4g). In Fig. 4h, it is revealed that the almost overlapping CV curves tested at $200 \mathrm{mV} \mathrm{s}^{1}$ from ?at to 180 evidenced the outstanding ?exibility of PMMSCs-EBIE. Furthermore, PMMSCs-EBIE can easily realize selfintegration to effectively boost the output voltage and capacitance through continuous one-by-one transfer without the requirement of metal-based interconnections (Fig. 1k and 1). For instance, the operating voltage for obtaining CV curves exhibited a monotonous increase from $3 \mathrm{~V}$ for one cell, $6 \mathrm{~V}$ for two-serially connected cells, and $9 \mathrm{~V}$ for three-serially connected cells (Fig. $4 \mathrm{i}$ ). More importantly, one PM-MSCs-EBIE can easily power a DICP logo display for more than 5 min aler charging for $150 \mathrm{~s}$ at $0.2 \mathrm{~mA} \mathrm{~cm}{ }^{2}$ (Fig. $4 \mathrm{j}$ ). Furthermore, the three serially-connected PM-MSCs-EBIE pack can readily light the four letters of DICP composed of 42 light-emitting diodes (LEDs) in both ?at (Fig. 4k) and bending (Fig. 4l) states, indicating the dramatic potential of the PM-MSCs-EBIE as a standalone micropower source for microelectronics.
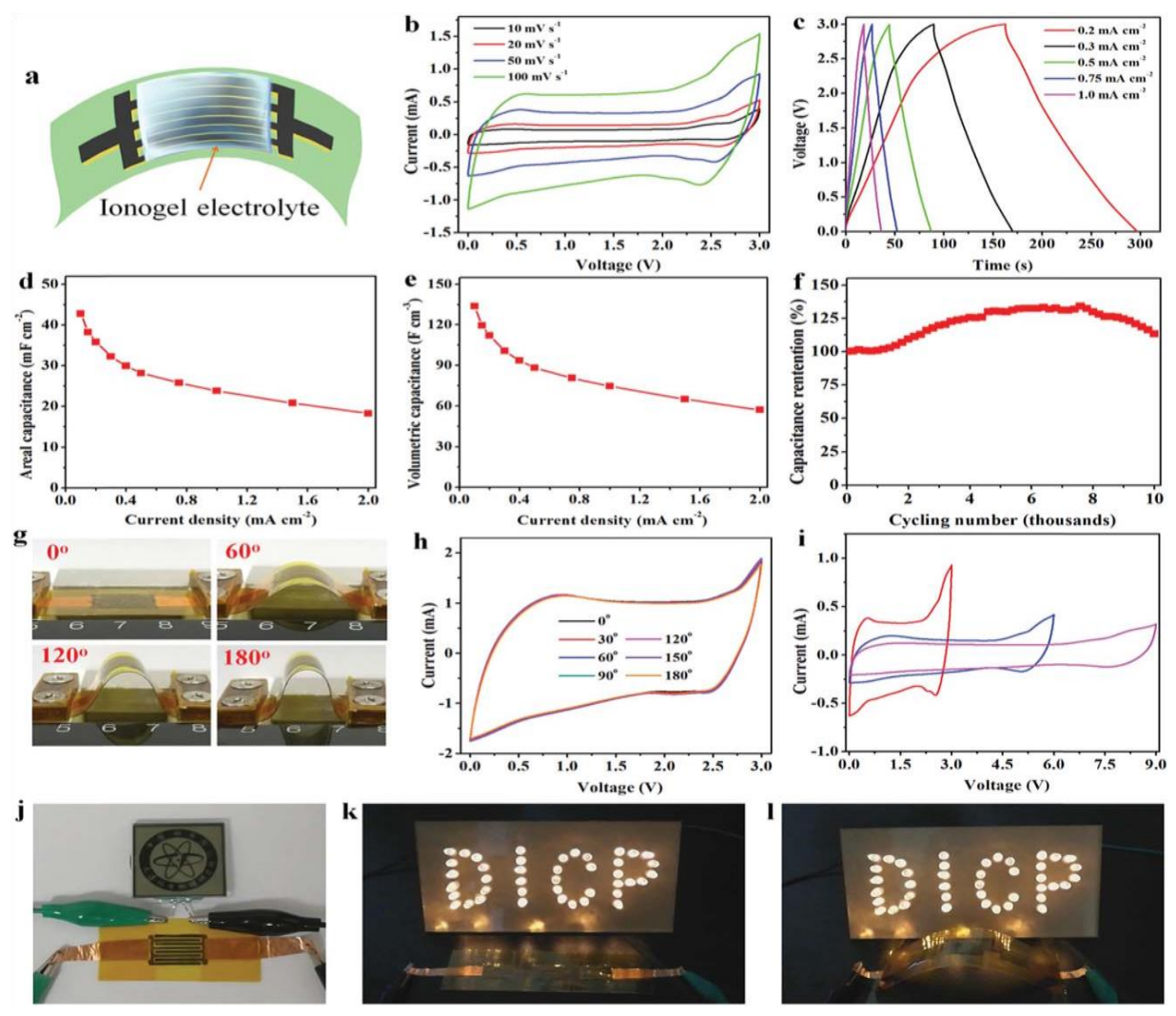

Fig. 4 Electrochemical performance of the all-solid-state PM-MSCs-EBIE. (a) Schematic of PM-MSCs-EBIE using the ionogel electrolyte. (b) CV curves tested from 10 to $100 \mathrm{mV} \mathrm{s}^{1}$. (c) GCD profiles measured from 0.2 to $1 \mathrm{~mA} \mathrm{~cm} \mathrm{~cm}^{2}$. (d) Areal capacitance and (e) volumetric capacitance obtained from 0.1 to $2 \mathrm{~mA} \mathrm{~cm}^{2}$. (f) Cyclability tested at $1.0 \mathrm{~mA} \mathrm{~cm}{ }^{2}$. (g) Optical images of flexible PM-MSCs-EBIE at various bending angles. (h) CV curves measured at different bending states at $200 \mathrm{mV} \mathrm{s}^{1}$. (i) CV curves of serially connected PM-MSCs-EBIE from 1 to 3 cells, tested at $50 \mathrm{mV} \mathrm{s}$. (j) Optical image of the DICP logo display powered by one PM-MSCs-EBIE. ( $k$ and $I$ ) Optical images of "DICP" letters composed of 42 LED lights, powered by three serially connected PM-MSCs-EBIE in flat ( $k$ ) and bending 
Ragone plots are shown in Fig. 5a and b to compare the areal and volumetric energy densities and power densities of PMMSCs in different ionic liquids with those of other previously reported M-MSCs and commercially available energy storage microdevices. Notably, PM-MSCsEB and PM-MSCs-EBIE offered high areal energy densities of 13.9 and $13.3 \mathrm{~mW} \mathrm{~h} \mathrm{~cm}{ }^{2}$, respectively, much higher than those of PM-MSCs-

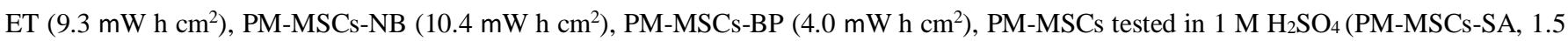

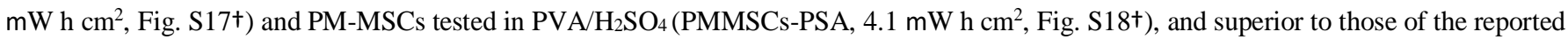
M-MSCs (Table S1 + ), such as MXene//Co-Al-layered double hydroxide (Co-Al-LDH) asymmetric MSCs $\left(10.8 \mathrm{~mW} \mathrm{~h} \mathrm{~cm}^{2}\right),{ }^{43} \mathrm{clay}-$ like MXene

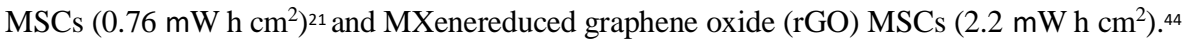

Moreover, PM-MSCs-EB also exhibited a high areal power density of $4.5 \mathrm{~mW} \mathrm{~cm}{ }^{2}$.

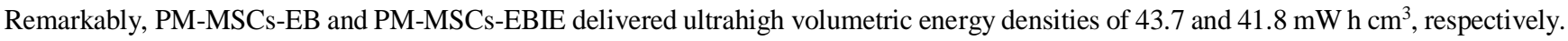

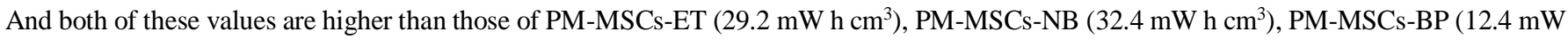
$\left.\mathrm{h} \mathrm{cm}^{3}\right)$, PM-MSCs-SA $\left(4.7 \mathrm{~mW} \mathrm{~h} \mathrm{~cm}^{3}\right)$, and PM-MSCs-PSA $\left(12.7 \mathrm{~mW} \mathrm{~h} \mathrm{~cm}^{3}\right)$, four times higher than those of lithium thin $\left.⿴ 囗 ⿱ 一 一\right) \mathrm{m}$ batteries $(<10$

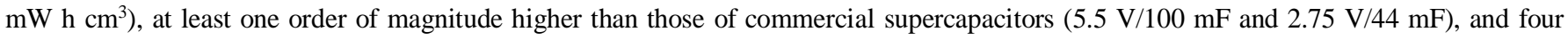
orders of magnitude higher than that of an $\mathrm{Al}$ electrolytic capacitor. ${ }^{17} \mathrm{It}$ is noteworthy that these values of 43.7 and $41.8 \mathrm{~mW} \mathrm{~h} \mathrm{~cm}^{3}$ are also $2-$

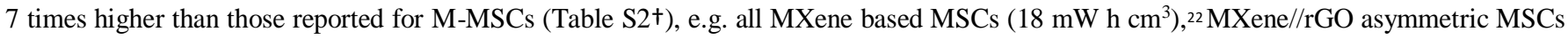

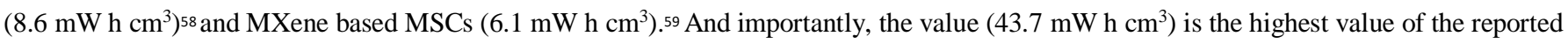
MMSCs to date. Furthermore, at a high power density of $14 \mathrm{~W} \mathrm{~cm}^{3}$, PM-MSCs-EB could still exhibit a considerable energy density of 19.2 $\mathrm{mWh} \mathrm{cm}^{3}$.

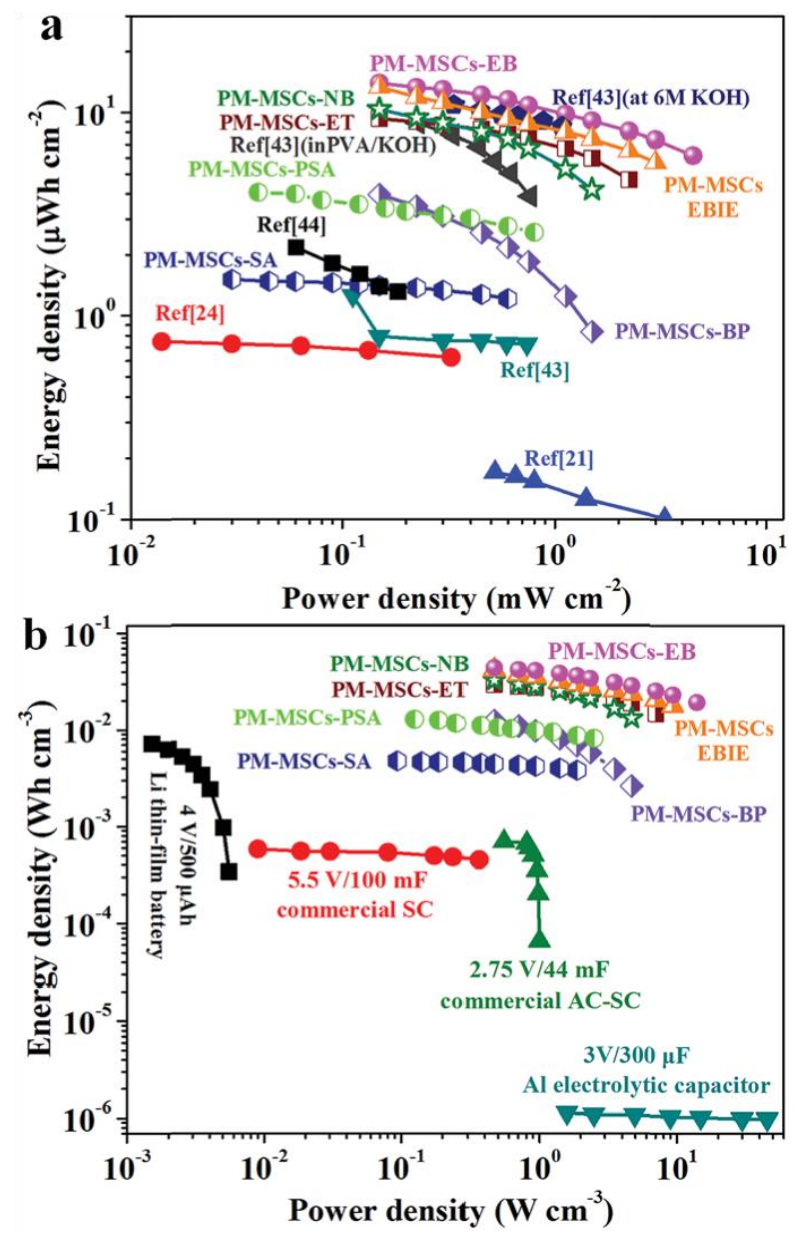

Fig. 5 Ragone plot to compare (a) areal and (b) volumetric energy density and power density of PM-MSCs in different electrolytes with other reported M-MSCs (MXene//Co-Al-LDH, ${ }^{43} \mathrm{Ti}_{3} \mathrm{C}_{2} \mathrm{Tx}$ hybrid ink, ${ }^{43}$

$\mathrm{Ti}_{3} \mathrm{C}_{2} \mathrm{Tx}_{\mathrm{ink}},^{24} \mathrm{Ti}_{3} \mathrm{C}_{2} \mathrm{Tx} /$ graphene aerogel, ${ }^{44}$ and $\mathrm{HF}$-etched $\left.\mathrm{Ti}_{3} \mathrm{C}_{2} \mathrm{~T}^{21}\right)$ and commercially available energy storage devices. 


\section{Conclusions}

In summary, we have demonstrated the successful construction of high-energy ionogel-based lexible and integrated MSCs using interdigital planar microelectrodes of ionic liquid preintercalated MXene alms, free of a metal current collector. The as-fabricated PM-MSCs with a $\mathrm{EMIMBF}_{4} / \mathrm{PVDF}-\mathrm{HFP}$ ionogel electrolyte delivered landmark areal and volumetric energy density. Moreover, all-solid-state PM-MSCs exhibited long-term stable cyclability up to 10000 cycles without capacitance fading, exceptional mechanical ?exibility, facile self-integration of bipolar cells and modularization for enhancing output voltage and capacitance. These ?ndings validate that the preintercalation of ionic liquid into 2D MXene compact [ [llms, with expanded interlayer spacing, can substantially provide extended nanospacing for enhanced accommodation of electrolyte ions and establishment of a continuous ion transport network, resulting in dramatic performance enhancement of MSCs. Considering a large family of MXenes and high volumetric capacitance (currently 100-200 $\mathrm{F} \mathrm{cm}^{3}$ in ionic liquids), this work will open up a new avenue for creating ionic liquid pre-intercalated MXene llms toward high-energy and ?exible MSCs.

\section{Conflicts of interest}

There are no con?icts to declare.

\section{Acknowledgements}

This work was ?nancially supported by the National Natural Science Foundation of China (Grants 51572259, 51872283 and 21805273), National Key R\&D Program of China (Grants 2016YFB0100100 and 2016YFA0200200), Natural Science Foundation of Liaoning Province (Grant 20180510038), DICP (DICP ZZBS201708 and DICP ZZBS201802), DICP\&QIBEBT (Grant DICP\&QIBEBT UN201702), Dalian National Laboratory For Clean Energy (DNL), CAS, DNL Cooperation Fund, CAS (DNL180310 and DNL180308), and Exploratory Research Program of Shaanxi Yanchang Petroleum (Group) CO., LTD \& DICP. VN would like to thank the support of the ERC CoG Grant 3D2DPrint

\section{Notes and references}

1 N. A. Kyeremateng, T. Brousse and D. Pech, Nat.

Nanotechnol., 2017, 12, 7-15.

2 Y. G. Wang, Y. F. Song and Y. Y. Xia, Chem. Soc. Rev., 2016, 45, 5925-5950.

3 K.-H. Choi, J. Yoo, C. K. Lee and S.-Y. Lee, Energy Environ. Sci., 2016, 9, 2812-2821.

4 S. Zheng, J. Ma, Z.-S. Wu, F. Zhou, Y. He, F. Kang, H.-M. Cheng and X. Bao, Energy Environ. Sci., 2018, 11, 2001-2009.

5 Z. T. Zhang, X. L. Chen, P. N. Chen, G. Z. Guan, L. B. Qiu, H. J. Lin, Z. B. Yang, W. Y. Bai, Y. F. Luo and H. S. Peng, Adv. Mater., 2014, 26, 466-470.

6 A. Sumboja, J. Liu, W. G. Zheng, Y. Zong, H. Zhang and Z. Liu, Chem. Soc. Rev., 2018, 47, 5919-5945. 7 Z.-S. Wu, X. Feng and H.-M. Cheng, Natl. Sci. Rev., 2014, 1, 277-292.

8 M. Beidaghi and Y. Gogotsi, Energy Environ. Sci., 2014, 7, 867-884.

9 D. Qi, Y. Liu, Z. Liu, L. Zhang and X. Chen, Adv. Mater., 2017, 29, 1602802.

10 J. Chmiola, C. Largeot, P. L. Taberna, P. Simon and

Y. Gogotsi, Science, 2010, 328, 480-483. 11 Z. S. Wu, K. Parvez, X. L. Feng and K. Mullen," Nat. Commun., 2013 , $4,2487$.

12 Z.-S. Wu, Y.-Z. Tan, S. Zheng, S. Wang, K. Parvez, J. Qin, X. Shi, C. Sun, X. Bao, X. Feng and K. Mullen,” J. Am. Chem. Soc., 2017, 139, 4506-4512.

13 J. Cai, C. Lv and A. Watanabe, Nano Energy, 2016, 30, 790- 800.

14 P. Huang, C. Lethien, S. Pinaud, K. Brousse, R. Laloo,

V. Turq, M. Respaud, A. Demorti`ere, B. Daffos, P. L. Taberna, B. Chaudret, Y. Gogotsi and P. Simon,

Science, 2016, 351, 691-695.

15 D. Pech, M. Brunet, H. Durou, P. H. Huang, V. Mochalin, Y. Gogotsi, P. L. Taberna and P. Simon, Nat. Nanotechnol., $2010,5,651-654$.

16 J. Lin, C. Zhang, Z. Yan, Y. Zhu, Z. Peng, R. H. Hauge, D. Natelson and J. M. Tour, Nano Lett., 2013, 13, 72-78.

17 M. F. El-Kady and R. B. Kaner, Nat. Commun., 2013, 4, 1475. 18 M. M. Wu, Y. R. Li, B. W. Yao, J. Chen, C. Li and G. Q. Shi, J. Mater. Chem. A, 2016, 4, 16213-16218.

19 F. Zhou, H. Huang, C. Xiao, S. Zheng, X. Shi, J. Qin, Q. Fu, X. Bao, X. Feng, K. Mullen and Z.-S. Wu,” J. Am. Chem. Soc., 2018, 140, 8198-8205.

20 X. Shi, S. Zheng, Z.-S. Wu and X. Bao, J. Energy Chem., 2018, 27, 25-42.

21 N. Kurra, B. Ahmed, Y. Gogotsi and H. N. Alshareef, Adv. Energy Mater., 2016, 6, 1601372. 
22 Y.-Y. Peng, B. Akuzum, N. Kurra, M.-Q. Zhao, M. Alhabeb,

B. Anasori, E. C. Kumbur, H. N. Alshareef, M.-D. Ger and

Y. Gogotsi, Energy Environ. Sci., 2016, 9, 2847-2854. 23 H. Li, Y. Hou, F. Wang, M. R. Lohe, X. Zhuang, L. Niu and X. Feng, Adv. Energy Mater., 2017, 7, 1601847. 24 C. Zhang, M. P. Kremer, A. Seral-Ascaso, S.-H. Park, N. McEvoy, B. Anasori, Y. Gogotsi and V. Nicolosi, Adv. Funct. Mater., 2018, 28, 1705506.

25 Z. S. Wu, K. Parvez, S. Li, S. Yang, Z. Y. Liu, S. H. Liu, X. L. Feng and K. Muellen, Adv. Mater., 2015, $27,4054-4061$.

26 Z.-S. Wu, Y. Zheng, S. Zheng, S. Wang, C. Sun, K. Parvez, T. Ikeda, X. Bao, K. Mullen and X. Feng," Adv. Mater., $2017,29,1602960$.

27 E. Eustache, C. Douard, R. Retoux, C. Lethien and

T. Brousse, Adv. Energy Mater., 2015, 5, 1500680. 28 Z. Li, M. Shao, L. Zhou, R. Zhang, C. Zhang, J. Han, M. Wei, D. G. Evans and X. Duan, Nano Energy, 2016, 20, 294-304.

29 J. J. Yoo, K. Balakrishnan, J. Huang, V. Meunier, B. G. Sumpter, A. Srivastava, M. Conway, A. L. Mohana Reddy, J. Yu, R. Vajtai and P. M. Ajayan, Nano Lett., 2011, 11, 1423-1427.

30 P. Zhang, F. Wang, M. Yu, X. Zhuang and X. Feng, Chem. Soc. Rev., 2018, 47, 7426-7451.

31 Y. Dong, S. Zheng, J. Qin, X. Zhao, H. Shi, X. Wang, J. Chen and Z.-S. Wu, ACS Nano, 2018, 12, 2381-2388. 32 N. K. Chaudhari, H.

Jin, B. Kim, D. S. Baek, S. H. Joo and K. Lee, J. Mater. Chem. A, 2017, 5, 24564-24579.

33 Q. Yang, T. P. Jiao, M. Li, Y. B. Li, L. T. Ma, F. N. Mo,

G. J. Liang, D. H. Wang, Z. F. Wang, Z. H. Ruan, W. J. Zhang, Q. Huang and C. Y. Zhi, J. Mater. Chem. A, 2018,6 , $18525-18532$.

34 M. R. Lukatskaya, O. Mashtalir, C. E. Ren, Y. Dall'Agnese,

P. Rozier, P. L. Taberna, M. Naguib, P. Simon, M. W. Barsoum and Y. Gogotsi, Science, 2013, 341, $1502-1505$.

35 X. Zhang, Z. H. Zhang and Z. Zhou, J. Energy Chem., 2018, 27, 73-85.

36 M. Lu, H. J. Li, W. J. Han, J. N. Chen, W. Shi, J. H. Wang, X. M. Meng, J. G. Qi, H. B. Li, B. S. Zhang, W. Zhang and

W. T. Zheng, J. Energy Chem., 2019, 31, 148-153.

37 A. VahidMohammadi, J. Moncada, H. Z. Chen, E. Kayali, J. Orangi, C. A. Carrero and M. Beidaghi, J. Mater. Chem. A, 2018, 6, 2212322133.

38 F. Wu, Y. Jiang, Z. Q. Ye, Y. X. Huang, Z. H. Wang, S. J. Li, Y. Mei, M. Xie, L. Li and R. J. Chen, J. Mater. Chem. A, 2019, 7, 13151322.

39 Y. Xia, T. S. Mathis, M.-Q. Zhao, B. Anasori, A. Dang, Z. Zhou, H. Cho, Y. Gogotsi and S. Yang, Nature, 2018, 557, 409-412.

40 A. D. Dillon, M. J. Ghidiu, A. L. Krick, J. Griggs, S. J. May, Y. Gogotsi, M. W. Barsoum and A. T. Fafarman, Adv. Funct. Mater., 2016, 26, 4162-4168.

41 M. R. Lukatskaya, S. Kota, Z. Lin, M.-Q. Zhao, N. Shpigel, M. D. Levi, J. Halim, P.-L. Taberna, M. W. Barsoum,

P. Simon and Y. Gogotsi, Nat. Energy, 2017, 6, 17105.

42 C. Zhang, B. Anasori, A. Seral-Ascaso, S.-H. Park, N. McEvoy,

A. Shmeliov, G. S. Duesberg, J. N. Coleman, Y. Gogotsi and

V. Nicolosi, Adv. Mater., 2017, 29, 1702678. 43 S. Xu, Y. Dall'Agnese, G. Wei, C. Zhang, Y. Gogotsi and W. Han, Nano Energy, 2018, 50, 479-488.

44 Y. Yue, N. Liu, Y. Ma, S. Wang, W. Liu, C. Luo, H. Zhang, F. Cheng, J. Rao, X. Hu, J. Su and Y. Gao, ACS Nano, $2018,12,4224-4232$. 45 Z. Lin, D. Barbara, P.-L. Taberna, K. L. Van Aken, B. Anasori, Y. Gogotsi and P. Simon, J. Power Sources, $2016,326,575-579$.

46 Z. Lin, P. Rozier, B. Duployer, P.-L. Taberna, B. Anasori, Y. Gogotsi and P. Simon, Electrochem. Commun., $2016,72,50-53$.

47 X. Yang, C. Cheng, Y. Wang, L. Qiu and D. Li, Science, 2013, 341, 534-537.

48 A. Lipatov, M. Alhabeb, M. R. Lukatskaya, A. Boson, Y. Gogotsi and A. Sinitskii, Adv. Electron. Mater., 2016,2 , 1600255.

49 F. Shahzad, M. Alhabeb, C. B. Hatter, B. Anasori, S. Man Hong, C. M. Koo and Y. Gogotsi, Science, 2016, 353, 1137- 1140.

50 N. Jackel, B. Kruner, K. L. Van Aken, M. Alhabeb, B. Anasor, F. Kaasik, Y. Gogotsi and V. Presser, ACS Appl. Mater. Interfaces, 2016, 8, 32089-32093.

51 Y. Zhang, R. Ye, D. Henkensmeier, R. Hempelmann and R. Chen, Electrochim. Acta, 2018, 263, 47-52. 52 J. Yan, C. E. Ren, K. Maleski,

C. B. Hatter, B. Anasori, P. Urbankowski, A. Sarycheva and Y. Gogotsi, Adv. Funct. Mater., 2017, $27,1701264$.

53 Y. Dall'Agnese, P. Rozier, P.-L. Taberna, Y. Gogotsi and P. Simon, J. Power Sources, 2016, 306, 510-515.

54 J. Fuller, R. T. Carlin and R. A. Osteryoung, J. Electrochem. Soc., 1997, 144, 3881-3886.

55 A. B. McEwen, H. L. Ngo, K. LeCompte and J. L. Goldman, J. Electrochem. Soc., 1999, 146, 1687-1695. 56 Z. Chen, S. Liu, Z. Li, Q.

Zhang and Y. Deng, New J. Chem., 2011, 35, 1596-1606.

57 J. A. Widegren, E. M. Saurer, K. N. Marsh and J. W. Magee, J. Chem. Thermodyn., 2005, 37, 569-575. 
58 C. Couly, M. Alhabeb, K. L. V. Aken, N. Kurra, L. Gomes, A. M. Navarro-Su'arez, B. Anasori, H. N. Alshareef and Y. Gogotsi, Adv. Electron. Mater., 2018, 4, 1700339.

59 H. B. Hu and T. Hua, J. Mater. Chem. A, 2017, 5, 19639-19648. 